\title{
Precision Calculations to Top- and Bottom-Yukawa Couplings within the SM and BSM
}

\author{
Luminita Mihaila* \\ Institut for Theoretical Physics, University of Heidelberg, 69117 Heidelberg, Germany \\ E-mail: mihaila@thphys.uni-heidelberg.de
}

\begin{abstract}
We report in this presentation on the recent calculations of the mixed QCD-EW corrections to the top- and bottom-Yukawa couplings within the Standard Model (SM) and its minimal supersymmetric extension (MSSM). We concentrate on the comparison between the SM and the MSSM predictions and on the non-decoupling behavior of the SUSY corrections that might open a new window to indirect searches for new physics.
\end{abstract}

The European Physical Society Conference on High Energy Physics

5-12 July, 2017

Venice

${ }^{*}$ Speaker. 


\section{Motivation}

After the end of the LHC run I and the start of the LHC run II, the direct searches for supersymmetric particles remained unsuccessful, thus increasing the exclusion bounds for the supersymmetric mass scale towards the $\mathrm{TeV}$ range. For the time being, it seems that if supersymmetry is realised in high energy physics the most likely scenario is the one where all SUSY particles are much heavier than the SM ones. For such a mass hierarchy (also called the "decoupling limit"), it was shown explicitly [1] that the effects of the SUSY particles on many physical observables in the gauge and Higgs sectors scales like $M_{\mathrm{EW}}^{2} / M_{\mathrm{SUSY}}^{2}$. This conclusion is just a consequence of the Appelquist-Carrazone decoupling theorem [2], that states that the effects of the heavy fields decouple at low momenta except for their contribution to renormalization effects. Therefore, for very heavy SUSY scales, deviations from the SM predictions for these observables will be very challenging to detect. In such a case, additional efforts both in theory and experiment are required to identify physical observables for which the power suppressed behaviour does not occur (i.e. the Appelquist-Carrazone theorem is violated), or the decoupling limit is delayed by parametric enhancements like in the presence of large $\tan \beta$ values [3]. Prominent examples for this class of observables are the mass of the lightest Higgs boson in SUSY and its self coupling in BSM theories $[4,5]$ that receive radiative corrections increasing with the heavy mass scales and their mixing parameters.

In the present study $[10,11]$ we consider the behaviour of the running top quark mass and Yukawa coupling in the decoupling limit of the MSSM. Under the decoupling limit we understand that all the superpartners, including the additional Higgs particles $A, H^{0}, H^{ \pm}$, are much heavier than the SM particles. We assume here that the relation $M_{\mathrm{SUSY}}, M_{\mathrm{A}} \gg M_{\mathrm{EW}}$ holds. The case for an intermediate Higgs sector ( $M_{\mathrm{SUSY}} \gg M_{\mathrm{A}} \gg M_{\mathrm{EW}}$ ), i.e at intermediate energies the 2HDM is at work, was studied in detail in the literature [3, 6, 7, 8, 9]. Here, we consider the case for which the lightest Higgs boson behaves SM like and no additional particles have masses at intermediate energy scales.

The non-decoupling effects for the running quark masses and Yukawa couplings increase logarithmically with the SUSY mass scale and have a polynomial dependence with maximal degree three on the mixing parameters in the squark sectors. From a phenomenological point of view, the non-decoupling effects are usually few times larger than the current accuracy on the bottom and top quark mass determinations. For Yukawa couplings the effects are in the range of precision expected for a $100 \mathrm{TeV}$ collider. In order to keep the theoretical uncertainties well below the experimental accuracy, we consider here the two-loop radiative corrections of order $\mathscr{O}\left(\alpha_{s}^{2}, \alpha_{s} \alpha_{t}\right)$ induced by the strong and the top-Yukawa couplings, and neglect the bottom-Yukawa coupling except for scenarios with large $\tan \beta$ values.

\section{Framework}

For the study of the running quark masses and Yukawa couplings up to $\mathscr{O}\left(\alpha_{s}^{2}, \alpha_{s} \alpha_{t}\right)$ it is sufficient to study the following three operators

$$
\mathscr{L}_{\mathrm{eff}}=\mathscr{L}_{\mathrm{SM}}^{(6)}+\mathscr{L}_{\mathrm{eff}}^{h}+\ldots ; \quad \mathscr{L}_{\mathrm{eff}}^{h}=-\frac{h^{(0)}}{v^{(0)}}\left[C_{1}^{0} \mathscr{O}_{1}^{0}+\sum_{q=u, d, \ldots}\left(C_{2 q}^{0} \mathscr{O}_{2 q}^{0}+C_{3 q}^{0} \mathscr{O}_{3 q}^{0}\right)+\ldots\right]
$$


Here $\mathscr{L}_{\mathrm{SM}}^{(6)}$ denotes the usual SM Lagrangian with six active quark flavours but without the Yukawa sector. The latter is considered explicitely in $\mathscr{L}_{\text {eff }}^{h}$, that collects all interactions with just one Higgs field. The ellipsis stand for the remaining higher-dimensional operators that we do not study here. The Wilson coefficients $C_{i}, i=1,2 q, 3 q$, parameterize the effects of the heavy particles and the superscript 0 labels bare quantities. The three operators relevant in our computation are defined as

$$
\begin{aligned}
\mathscr{O}_{1}^{0} & =\left(G_{\mu, v}^{0, \prime a}\right)^{2} \\
\mathscr{O}_{2 q}^{0} & =m_{q}^{0, \bar{q}^{0, \prime}} q^{0, \prime} \\
\mathscr{O}_{3 q}^{0} & =\bar{q}^{0, \prime}\left(i p^{0, \prime}-m_{q}^{0, \prime}\right) q^{0, \prime},
\end{aligned}
$$

where $G_{\mu, v}^{0,, a}$ and $D_{\mu}^{0, \prime}$ are the gluon field strength tensor and the covariant derivative for quark fields, and the primes label the quantities in the effective theory. The operator $\mathscr{O}_{3 q}$ vanishes by the fermionic equation of motion and it will not contribute to physical observables. So, the last term in Eq. (2.1) might be omitted, once the coefficients $C_{1}^{0}, C_{2 q}^{0}$ are determined. In the decoupling limit $C_{1} \rightarrow 0$ and $C_{2 q} \rightarrow 1$ and the higher-dimensional operators decouple. We verified through an explicit calculation that these relations hold at one and two loops in the top sector.

Furthermore, one has to perform a redefinition of the parameters and fields present in the EFT. In mass independent renormalization schemes the Appelquist-Carrazone decoupling theorem does not hold and the Green functions usually depend on the heavy masses and mixing parameters present in the full theory. In order to avoid potentially large logarithms and power corrections one has to decouple the heavy particles from the theory. As a consequence, one obtains different Lagrangian parameters in the various energy regimes. They are connected by the so-called decoupling or matching relations that can be computed order by order in perturbation theory. We computed the decoupling coefficients for the quark masses and the third generation Yukawa-coupling at $\mathscr{O}\left(\alpha_{s}^{2}, \alpha_{s} \alpha_{t}\right)$.

\section{Results}

In this section, we study the phenomenological implications of the two-loop calculation of the decoupling coefficients. Two-loop decoupling effects are usually combined with three-loop renormalization group equations (RGEs) in order to properly resum the logarithms that arise in the calculation.

We show in Fig. 1 the scale evolution of the three parameters both in the SM and the MSSM for the pMSSM scenario, where the matching between the two theories was performed at $\mu_{\mathrm{dec}}=$ $400 \mathrm{GeV}$. Namely, below $400 \mathrm{GeV}$ the SM is considered as the theory describing the physical phenomena. Above this energy scale, the MSSM is the underlying theory. For the dot-dashed lines, only the QCD (below $\mu_{\mathrm{dec}}=400 \mathrm{GeV}$ ) and SUSY-QCD (above $\mu_{\mathrm{dec}}=400 \mathrm{GeV}$ ) contributions are taken into account. For the one-loop running (dotted lines in the plots) only a change of slopes occurs, because no decoupling is taken into account. At two and three loops, the running is accompanied by one- and two-loop decoupling. The vertical shifts at $\mu_{\mathrm{ren}}=\mu_{\mathrm{dec}}=400 \mathrm{GeV}$ display directly the effects of the decoupling procedure. The numerical values of the mixed (SUSY)QCD-Yukawa contributions at three loops can be read from the difference between the full and the dot-dashed curves in the figure. As already pointed out, their magnitude depends on the scale choice and are 


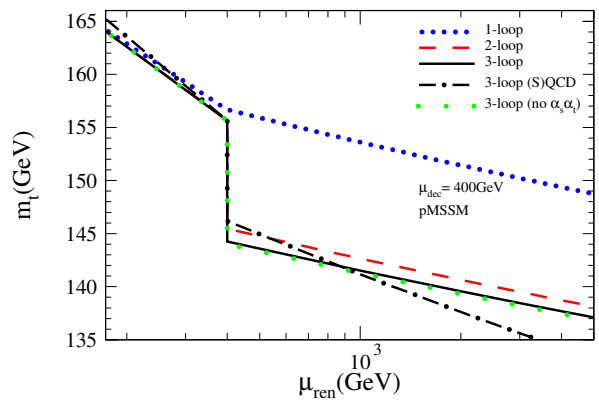

(a)

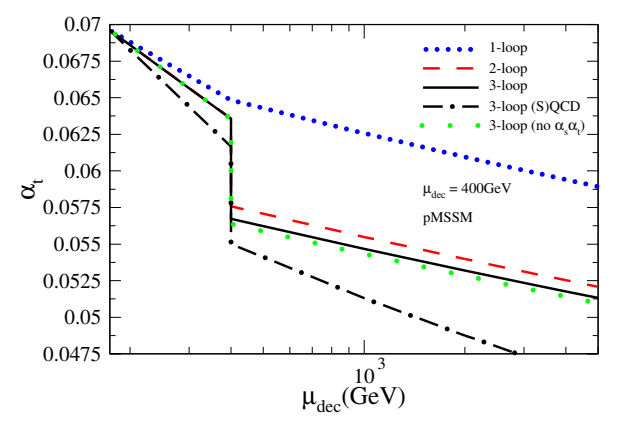

(b)

Figure 1: Running of $m_{t}$ and $\alpha_{t}$. The matching scale between the SM and the MSSM is $\mu_{\mathrm{dec}}=$ $400 \mathrm{GeV}$. The dotted, dashed and full lines correspond to one-, two- and three-loop running. The dot-dashed lines display the three-loop SUSY-QCD contributions. The large dotted lines marked in the legend with the label (no $\alpha_{s} \alpha_{t}$ ) show the predictions of the three-loop analysis, where the $\mathscr{O}\left(\alpha_{s} \alpha_{t}\right)$ contributions to the decoupling coefficients for $m_{t}$ and $\alpha_{t}$ were excluded. The jumps at $\mu_{\mathrm{dec}}=400$ display the decoupling effects.

phenomenologically significant for the top sector of the MSSM. The magnitude of the $\mathscr{O}\left(\alpha_{s} \alpha_{t}\right)$ corrections to the decoupling coefficients for $m_{t}$ and $\alpha_{t}$ can be read from the difference between the solid and the large dotted lines. For the MSSM scenario under consideration, they are much smaller than the expected experimental precision.

Although the running masses and couplings are themselves not physical observables, they are necessary ingredients for the theoretical predictions of cross sections, branching ratios or physical masses. For example, if in a diagrammatic calculation performed within the MSSM at fixed order or in the EFT approach the running top-quark mass and/or running top-Yukawa and strong couplings are used, then the non-decoupling effects we discussed here are implicitly contained in the numerical values of the running parameters. From our numerical analysis it turned out that the genuine $\mathscr{O}\left(\alpha_{s} \alpha_{t}\right)$ contributions to the matching coefficients are well below the experimental precision. However, the complete $\mathscr{O}\left(\alpha_{t}, \alpha_{s} \alpha_{t}\right)$ corrections (including the RGE and the threshold effects) to the running top quark mass amount to about few $\mathrm{GeV}$ and for the top-Yukawa coupling reach the percent range. The $\mathscr{O}\left(\alpha_{s} \alpha_{t}\right)$ corrections to the decoupling coefficients for $m_{t}, \alpha_{s}$ and $\alpha_{t}$ are necessary, for example, for the prediction of the lightest Higgs boson mass in supersymmetric theories and for the vacuum stability studies in such theories, when going beyond two-loop accuracy.

\section{References}

[1] A. Dobado, M. J. Herrero and S. Penaranda, The Higgs sector of the MSSM in the decoupling limit, Eur. Phys. J. C 17 (2000) 487 [hep-ph / 0002134 ]; H. E. Haber, M. J. Herrero, H. E. Logan, S. Penaranda, S. Rigolin and D. Temes, Decoupling properties of MSSM particles in Higgs and top decays, [hep-ph/0102169].

[2] T. Appelquist and J. Carazzone, nfrared Singularities and Massive Fields, Phys. Rev. D 11 (1975) 2856. 
[3] L. J. Hall, R. Rattazzi and U. Sarid, The Top quark mass in supersymmetric SO(10) unification Phys. Rev. D 50 (1994) 7048; M. S. Carena, D. Garcia, U. Nierste and C. E. M. Wagner, Effective Lagrangian for the $\bar{t} b H^{+}$interaction in the MSSM and charged Higgs phenomenology Nucl. Phys. B 577 (2000) 88 [arXiv: hep-ph/9912516].

[4] P. H. Chankowski, S. Pokorski and J. Wagner, (Non)decoupling of the Higgs triplet effects, Eur. Phys. J. C 50 (2007) 919 [hep-ph/0605302].

[5] S. Kanemura, M. Kikuchi and K. Yagyu, Fingerprinting the extended Higgs sector using one-loop corrected Higgs boson couplings and future precision measurements, Nucl. Phys. B 896 (2015) 80 [arXiv:1502.07716 [hep-ph]].

[6] D. Noth and M. Spira, Supersymmetric Higgs Yukawa Couplings to Bottom Quarks at next-to-next-to-leading Order, JHEP 1106 (2011) 084 [arXiv: 1001.1935 [ hep-ph] ]; D. Noth and M. Spira, Higgs Boson Couplings to Bottom Quarks: Two-Loop Supersymmetry-QCD Corrections, Phys. Rev. Lett. 101 (2008) 181801 [arXiv:0808.0087 [hep-ph] ].

[7] L. Mihaila and C. Reisser, $O\left(\alpha_{s}^{2}\right)$ corrections to fermionic Higgs decays in the MSSM, JHEP 1008 (2010) 021 [arXiv: 1007.0693 [hep-ph]].

[8] R. Harlander, L. Mihaila and M. Steinhauser, Two-loop matching coefficients for the strong coupling in the MSSM, Phys. Rev. D 72 (2005) 095009; [arXiv: hep-ph / 05090 48]; A. V. Bednyakov, Running mass of the b-quark in QCD and SUSY QCD, Int. J. Mod. Phys. A 22 (2007) 5245 [arXiv:0707.0650 [hep-ph] ]; A. Bauer, L. Mihaila and J. Salomon, Matching coefficients for $\alpha_{s}$ and $m(b)$ to $O\left(\alpha_{s}^{2}\right)$ in the MSSM, JHEP 0902 (2009) 037 [arXiv: 0810.5101 [hep-ph]].

[9] A. V. Bednyakov, On the two-loop decoupling corrections to tau-lepton and b-quark running masses in the MSSM, Int. J. Mod. Phys. A 25 (2010) 2437 [arXiv:0912. 4652 [hep-ph] ].

[10] D. Kunz, L. Mihaila and N. Zerf, $\mathscr{O}\left(\alpha_{S}^{2}\right)$ corrections to the running top-Yukawa coupling and the mass of the lightest Higgs boson in the MSSM, JHEP 1412 (2014) 136 [arXiv: 1409.2297 [hep-ph]].

[11] L. Mihaila and N. Zerf, $\mathscr{O}\left(\alpha_{s} \alpha_{t}\right)$ (non)decoupling effects within the top-sector of the MSSM, JHEP 1705 (2017) 019 [arXiv:1612.06619 [hep-ph] ]. 